\title{
PERMUTATIONS AND PRESENTATIONS
}

\author{
PETER CHOLAK AND ROD DOWNEY
}

(Communicated by Andreas R. Blass)

\begin{abstract}
We say that an automorphism $\Phi$ of $\mathscr{E}^{*}$ (the lattice of recursively enumerable sets modulo the finite sets) is induced by a permutation $p$ iff for all $e, \Phi\left(W_{e}\right)={ }^{*} p\left(W_{e}\right)$. A permutation $h$ is called a presentation of $\Phi$ iff for all $e, \Phi\left(W_{e}\right)={ }^{*} W_{h(e)}$. In this paper, we will explore the degree-theoretic connections between these two notions. Using a new proof of the well-known fact that every automorphism is induced by a permutation $p$, we show that such a $p$ can be found recursively in $h \oplus \varnothing^{\prime \prime}$, where $h$ is a presentation of $\Phi$. The main result of the paper is to show that there is an effective automorphism of $\mathscr{E}^{*}$ which is not induced by a $\Delta_{2}$-permutation.
\end{abstract}

Ever since the ground-breaking paper of Post [Po], a central object of recursion theory has been the lattice of recursively enumerable sets modulo the finite sets, $\mathscr{E}^{*}$. This object, its automorphism group $\operatorname{Aut}\left(\mathscr{E}^{*}\right)$, and their relation to the degrees of unsolvability have been extensively studied.

In this paper we study the relationship between $\operatorname{Aut}\left(\mathscr{E}^{*}\right)$ and the group of permutations of $\omega$. It has long been known (see [Sol]) that each automorphism $\Phi$ of $\mathscr{E}^{*}$ is induced by a permutation $p$ of $\omega$. Our concern is the relationship between the complexity of $\Phi$ and the permutation that induces $\Phi$.

To be precise, we say $\Phi$ is a $\Delta_{n+1}$-automorphism iff $\Phi$ has a presentation $h$ ( $h$ is a permutation and for all $\left.e, \Phi\left(W_{e}\right)={ }^{*} W_{h(e)}\right)$ such that $h \leq_{T} \varnothing^{n}$. Furthermore, we say $\Phi$ is effective iff $\Phi$ has a recursive presentation. In this paper, we will examine whether every $\Delta_{n}$-automorphism can be induced by a $\Delta_{n}$-permutation.

In Theorem 1, we show that every automorphism is induced by a permutation $p$, where $p \leq_{T} h \oplus \varnothing^{\prime \prime}$ and $h$ is a presentation of $\Phi$. This slightly improves Soare's result (see [So1] or [So2, XV.2.5]) that every automorphism is induced by a permutation $p$, where $p \leq_{T}\left(h \oplus \varnothing^{\prime}\right)^{\prime}$ and $h$ is a presentation of $\Phi$.

Received by the editors October 7, 1991 and, in revised form, March 15, 1993.

1991 Mathematics Subject Classification. Primary 03D25.

Key words and phrases. Permutations, presentations, automorphism, recursively enumerable.

This research was started while the first author was a student associate and the second author was a member of the Mathematical Sciences Research Institute (MSRI). It was completed at Victoria University of Wellington (VUW). The first author was partially supported by a U.S. Department of Education Fellowship and VUW Postdoctoral Fellowship. The second author was partially supported by a VUW IGC grant, a U.S. / N.Z. binational grant, and the MSRI. The authors thank Michael Stob for his help. 
Therefore, if $\Phi$ is a $\Delta_{n}$-automorphism, for any $n \geq 3$, then $\Phi$ is induced by a $\Delta_{n}$-permutation.

By Jockusch (see [So2, XV.2.13]) we know that if $\Phi$ has a presentation whose degree is less than or equal to $0^{\prime}$ then $\Phi$ has a recursive presentation and hence there is no properly $\Delta_{2}$-automorphism.

Thus, the only question left open is the relationship between an effective automorphism and the complexity of the permutation that induces it. Martin (see [So2, XV.3]) showed that there are effective automorphisms which are not induced by any recursive permutation. There are many proofs of this result. For example, by [CDS] we know that if $A$ is incomplete, promptly simple, and not hyperhypersimple, then $A$ is effectively automorphic to a complete set and clearly such an automorphism cannot be induced by a recursive permutation $\left(A \equiv_{T} p(A)\right)$, if $p$ is recursive. In Theorem 2, we improve Martin's result to show: there is an effective automorphism of $\mathscr{E}^{*}$ which is not induced by a $\Delta_{2}$-permutation.

Our notation is standard and follows Soare [So2]. All sets used are r.e.

Theorem 1. Every automorphism $\Phi$ is induced by a permutation $p$, where $p$ $\leq_{T} h \oplus \varnothing^{\prime \prime}$ and $h$ is a presentation of $\Phi$.

Proof. Let $p=\bigcup p_{n}$, where finite partial functions $p_{n}$ are defined by induction on $n$ as follows: Let $p_{-1}=\varnothing$. Given any finite $L \subset \omega$ and any linear order $\prec$ on $L$, let $\sigma(L, x)=\left\{i: x \in W_{l_{i}}\right\}$, where $L=\left\{l_{0} \prec l_{1} \prec \ldots \prec l_{m}\right\}$ (So for all $x, \sigma(\varnothing, x)=\varnothing$.) $\sigma(L, x)$ is uniformly recursive (in indices for both $L$ and $\prec)$ in $\varnothing^{\prime}$. Since $\Phi$ is an automorphism, for any $e$ and any subset $\sigma$ of $\{0,1, \ldots, e\},\{x: \sigma(\{0 \prec 1 \prec \ldots \prec e\}, x)=\sigma\}$ is infinite iff $\{x: \sigma(\{h(0) \prec h(1) \prec \cdots \prec h(e)\}, x)=\sigma\}$ is infinite. Let $L \uparrow-1=\varnothing$, and let $L \mid i=\left\{l_{0} \prec l_{1} \prec \cdots \prec l_{i}\right\}$, where $L=\left\{l_{0} \prec l_{1} \prec \cdots \prec l_{m}\right\}$ and $i \leq m$.

For all even $n$, use the following procedure: Let $x_{n}$ be the least integer not in the domain of $p_{n-1}$. Using $h$, let $L_{n}=\left\{0 \prec 1 \prec \cdots \prec x_{n}\right\}$ and $\hat{L}_{n}=\left\{h(0) \prec h(1) \prec \cdots \prec h\left(x_{n}\right)\right\}$. Using $\varnothing^{\prime \prime}$, let $e_{n}$ be the greatest $e$ such that $-1 \leq e \leq x_{n}$ and there exists a $y$ not in the range of $p_{n-1}$ with $\sigma\left(L_{n} \mid e, x_{n}\right)=\sigma\left(\hat{\hat{L}}_{n} \mid e, y\right)$. Let $y_{n}$ be the least integer not in the range of $p_{n-1}$ such that $\sigma\left(L_{n} \mid e_{n}, x_{n}\right)=\sigma\left(\hat{L}_{n} \mid e_{n}, y_{n}\right)$. Define $p_{n}=p_{n-1} \bigcup\left\{\left(x_{n}, y_{n}\right)\right\}$.

For all odd $n$, use the following procedure: Let $y_{n}$ be the least integer not in the range of $p_{n-1}$. Using $h$, let $L_{n}=\left\{0 \prec 1 \prec \cdots \prec y_{n}\right\}$ and $\hat{L}_{n}=\left\{h(0) \prec h(1) \prec \cdots \prec h\left(y_{n}\right)\right\}$. Using $\varnothing^{\prime \prime}$, let $e_{n}$ be the greatest $e$ such that $-1 \leq e \leq y_{n}$ and there exists an $x$ not in the domain of $p_{n-1}$ with $\sigma\left(L_{n} \mid e, x\right)=\sigma\left(\hat{L}_{n} \mid e, y_{n}\right)$. Let $x_{n}$ be the least integer not in the domain of $p_{n-1}$ such that $\sigma\left(L_{n} \mid e_{n}, x_{n}\right)=\sigma\left(\hat{L}_{n} \mid e_{n}, y_{n}\right)$. Define $p_{n}=p_{n-1} \bigcup\left\{\left(x_{n}, y_{n}\right)\right\}$.

Clearly, $p=\bigcup p_{n}$ is a permutation and $p \leq_{T} h \oplus \varnothing^{\prime \prime}$. Since $\Phi$ is an automorphism of $\mathscr{E}^{*}, \lim _{n} e_{n}=\infty$, and hence, it follows that $p\left(W_{n}\right)={ }^{*}$ $W_{h(n)}$.

Theorem 2. There is an effective automorphism $\Phi$ of $\mathscr{E}^{*}$ such that $\Phi$ is not induced by any permutation whose Turing degree is less than or equal to $0^{\prime}$.

Proof. Building an effective automorphism of $\mathscr{E}^{*}$ is a complex task. We will fix two copies of the natural numbers $\omega$ and $\hat{\omega}$ (all integers living on the hatted side will wear hats). Now, given an enumeration of $\left\{W_{e}\right\}_{e<\omega}$ living in $\omega$, say $\left\{U_{n, s}\right\}_{n, s<\omega}$, we will build the image of $U_{n}$ in $\hat{\omega}, \hat{U}_{n}=\Phi\left(U_{n}\right)$. 
Given another enumeration of $\left\{W_{e}\right\}_{e<\omega}$ living in $\hat{\omega}$, say $\left\{V_{n, s}\right\}_{n, s<\omega}$, we will build the preimage of $V_{n}$ in $\omega, \hat{V}_{n}=\Phi^{-1}\left(V_{n}\right)$. To ensure that $\Phi$ is an automorphism of $\mathscr{E}^{*}$ we need to meet the requirements $R_{\nu}$ below. However, to state these requirements, we need the following definitions.

Definition 3. For any $e$, if we are given uniformly recursive enumerations $\left\{X_{n, s}\right\}_{n \leq e, s<\omega}$ and $\left\{Y_{n, s}\right\}_{n \leq e, s<\omega}$ of r.e. sets $\left\{X_{n}\right\}_{n \leq e}$ and $\left\{Y_{n}\right\}_{n \leq e}$, define the full e-state of $x$ at stage $s, \nu(e, x, s)$, with respect to (w.r.t.) $\left\{\bar{X}_{n, s}\right\}_{n, s<\omega}$ and $\left\{Y_{n, s}\right\}_{n, s<\omega}$ to be the triple

$$
\nu(e, x, s)=\langle e, \sigma(e, x, s), \tau(e, x, s)\rangle
$$

where

$$
\sigma(e, x, s)=\left\{i: i \leq e \wedge x \in X_{i, s}\right\} \text { and } \tau(e, x, s)=\left\{i: i \leq e \wedge x \in Y_{i, s}\right\} .
$$

Definition 4. Given any collection of r.e. sets $\left\{X_{n}\right\}_{n<\omega}$ and $\left\{Y_{n}\right\}_{n<\omega}$, define the final $e$-state of $x, \nu(e, x)$, with respect to $\left\{X_{n}\right\}_{n<\omega}$ and $\left\{Y_{n}\right\}_{n<\omega}$ to be the triple

$$
\nu(e, x)=\langle e, \sigma(e, x), \tau(e, x)\rangle
$$

where

$$
\sigma(e, x)=\left\{i: i \leq e \wedge x \in X_{i}\right\} \text { and } \tau(e, x)=\left\{i: i \leq e \wedge x \in Y_{i}\right\} .
$$

(This last definition is slightly different from the normal definition of the final e-state of $x$. Normally, $\lim _{s \rightarrow \infty} \nu(e, x, s)=\nu(e, x)$ But that assumes that there are uniformly recursive enumerations of $\left\{X_{n}\right\}_{n<\omega}$ and $\left\{Y_{n}\right\}_{n<\omega}$. In this construction, that will be the case, but it is not always the case; see [Ch].)

Now to ensure that $\Phi$ is an automorphism of $\mathscr{E}^{*}$, where $\Phi\left(U_{n}\right)=\hat{U}_{n}$ and $\Phi^{-1}\left(V_{n}\right)=\hat{V}_{n}$, we need to meet the following requirements: for all $n$,

$\left(Q_{n}\right) \quad$ both $\hat{U}_{n}$ and $\hat{V}_{n}$ are r.e.

and for each final $e$-state $\nu$,

$\exists^{\infty} x \in \omega$ with final $e$-state $\nu$ w.r.t. to $\left\{U_{n}\right\}_{n<\omega}$ and $\left\{\hat{V}_{n}\right\}_{n<\omega}$

iff

$\exists^{\infty} \hat{x} \in \hat{\omega}$ with final $e$-state $\nu$ w.r.t. to $\left\{\hat{U}_{n}\right\}_{n<\omega}$ and $\left\{V_{n}\right\}_{n<\omega}$.

To ensure $\Phi$ is an effective automorphism, we must build $U_{n}, V_{n}, \hat{U}_{n}$, and $\hat{V}_{n}$ such that for some recursive functions $f_{1}$ and $f_{2}, W_{e}={ }^{*} U_{f_{1}(e)}$ and $\hat{U}_{n}={ }^{*} W_{f_{2}(n)}$ (i.e., $\left.\Phi\left(W_{e}\right)={ }^{*} W_{f_{2}\left(f_{1}(e)\right)}\right)$ and for some recursive functions $g_{1}$ and $g_{2}, W_{e}=V_{g_{1}(e)}$ and $\hat{V}_{n}={ }^{*} W_{g_{2}(n)}$ (i.e., $\left.\Phi^{-1}\left(W_{e}\right)={ }^{*} W_{g_{2}\left(g_{1}(e)\right)}\right)$. (Given $f_{i}$ and $g_{i}$, by using the Padding Lemma (see [So1, I.3.2]), we can construct a recursive permutation $h$ such that $\Phi\left(W_{n}\right)=\hat{U}_{n}={ }^{*} W_{h(n)}$ and hence $\Phi$ is an effective automorphism.)

Let $p$ be a permutation of $\omega$. If $\Phi$ is induced by $p^{-1}$ then for all r.e. sets $X, \Phi(X)={ }^{*} p^{-1}(X)$. We will think of $p^{-1}$ as a one-to-one onto map from $\omega$ to $\hat{\omega}$. Hence, to show $\Phi$ is not induced by $p^{-1}$ (also a permutation of $\operatorname{deg}(p))$, it is enough to either find some r.e. set $X$ and infinitely many $\hat{x}_{i}$ such that

$$
\left.\forall i\left[\hat{x}_{i} \notin \Phi(X) \text { but } \hat{x}_{i} \in p^{-1}(X) \quad \text { (or equivalently } p\left(\hat{x}_{i}\right) \in X\right)\right]
$$


or to find some r.e. set $Y$ and infinitely many $\hat{x}_{i}$ such that

$$
\left.\forall i\left[\hat{x}_{i} \in \Phi(Y) \text { but } \hat{x}_{i} \notin p^{-1}(Y) \quad \text { (or equivalently } p\left(\hat{x}_{i}\right) \notin Y\right)\right] \text {. }
$$

By the Limit Lemma, if $\operatorname{deg}(p) \leq_{T} 0^{\prime}$, there exists an $e$ such that for all $\hat{x}$, $p(\hat{x})=\lim _{s \rightarrow \omega}\{e\}(\hat{x}, s)$. Therefore, to ensure that $\Phi$ is not induced by a $\Delta_{2}^{0}$-permutation, it is enough to meet the following requirements:

$\lambda \hat{x} \lim _{s \rightarrow \omega}\{e\}(\hat{x}, s)$ is not a permutation; or

there is an r.e. set $X_{e}$ and infinitely many $\hat{x}_{e, i}$ such that

$$
\left.\forall i\left[\hat{x}_{e, i} \notin \Phi\left(X_{e}\right) \text { but } \lim _{s \rightarrow \omega}\{e\}\left(\hat{x}_{e, i}, s\right) \in X_{e}\right)\right] \text {; or }
$$

there is an r.e. set $Y_{e}$ and infinitely many $\hat{x}_{e, i}$ such that

$$
\left.\forall i\left[\hat{x}_{e, i} \in \Phi\left(Y_{e}\right) \text { but } \lim _{s \rightarrow \omega}\{e\}\left(\hat{x}_{e, i}, s\right) \notin Y_{e}\right)\right] \text {. }
$$

The easiest strategy to meet this requirement is for each $e$ to choose infinitely many $\hat{x}_{e, i}$ and for all $i, s$, and $t$ if $\{e\}_{t}\left(\hat{x}_{e, i}, s\right) \downarrow$ place $\{e\}_{t}\left(\hat{x}_{e, i}, s\right)$ into $X_{e}$ but hold $\hat{x}_{e, i}$ out of $\Phi\left(X_{e}\right)$. Unfortunately, we cannot do this and meet $\left(R_{\nu}\right)$. For example, it is possible under this strategy that $X_{e}=\omega$ (this could occur if for all $\left.i,\{e\}_{i}\left(\hat{x}_{e, i}, i\right) \downarrow=i\right)$. However, by modifying the above strategy as follows it is possible to meet $\left(R_{\nu}\right)$ and $\left(P_{e}\right)$.

Since we are allowed to choose any enumeration of $\left\{W_{e}\right\}_{e<\omega}$, we can let $U_{2 i, s}=X_{i, s}$ and $U_{2 i+1, s}=W_{i, s}$. This is, we will build $U_{2 e}$ during the construction to witness the meeting of requirement $\left(P_{e}\right)$. Note that this gives us control over $\Phi\left(X_{e}\right)=\Phi\left(U_{2 e}\right)$. Let $\left\{M_{i}: i<\omega\right\}$ be a uniformly recursive collection of disjoint infinite recursive sets such that for all $x$ there exists an $i$ with $x \in M_{i}\left(\hat{M}_{i}\right.$ will be a copy of $M_{i}$ in $\left.\hat{\omega}\right)$. Let $V_{2 i, s}=\hat{M}_{i, s}$ and $V_{2 i+1, s}=W_{i, s}$. (Hence, recursive $f_{1}$ and $g_{1}$ exist.)

Hence, we can meet $\left(P_{e}\right)$ by meeting the following subrequirements:

$\lambda \hat{x} \lim _{s \rightarrow \omega}\{e\}(\hat{x}, s)$ is not a permutation; or

there is an $\hat{x}_{e, k} \geq k$ such that $\hat{x}_{e, k} \in V_{2 e}$ and $\hat{x}_{e, k} \notin \Phi\left(U_{2 e}\right)$

$$
\text { but either } \lim _{s \rightarrow \omega}\{e\}\left(\hat{x}_{e, k}, s\right) \notin \hat{V}_{2 e} \text { or } \lim _{s \rightarrow \omega}\{e\}\left(\hat{x}_{e, k}, s\right) \in U_{2 e} \text {. }
$$

If we build $\Phi$ to be an automorphism and meet the subrequirement $\left(P_{e, k}\right)$ for all $k$, then we will have met $\left(P_{e}\right)$. Either there are infinitely many $\hat{x}$ such that $\hat{x} \notin \Phi\left(U_{2 e}\right)$ and $\lim _{s \rightarrow \omega}\{e\}(\hat{x}, s) \in U_{2 e}$, or there are infinitely many $\hat{x}$ such that $\hat{x} \in V_{2 e}={ }^{*} \Phi\left(\hat{V}_{2 e}\right)$ and $\lim _{s \rightarrow \omega}\{e\}(\hat{x}, s) \notin \hat{V}_{2 e}$. We will meet the subrequirement $\left(P_{e, k}\right)$ (and hopefully $\left(R_{\nu}\right)$ as well) as follows:

At each stage $s$, we will associate with $\left(P_{e, k}\right)$ two finite sets $F_{e, k, s}$ (a subset of $M_{e}$ ) and $\hat{F}_{e, k, s}$ (a subset of $\hat{M}_{e}$ ). We will ensure that $\bigcup\left\{F_{e, k, 0}: k<\omega\right\}=$ $M_{e}, \bigcup\left\{\hat{F}_{e, k, 0}: k<\omega\right\}=\hat{M}_{e}$, and if $k_{0} \neq k_{1}$ then $F_{e, k_{0}, 0} \cap F_{e, k_{1}, 0}=\varnothing$ and $\hat{F}_{e, k_{0}, 0} \cap \hat{F}_{e, k_{1}, 0}=\varnothing$ We will try to ensure that $F_{e, k, s} \supseteq F_{e, k, s+1}, \hat{F}_{e, k, s} \supseteq$ $\hat{F}_{e, k, s+1}, \hat{F}_{e, k, s} \cap \hat{U}_{2 e, s}=\varnothing$,

$$
\left|\hat{F}_{e, k, s}\right|>\left|\bigcup\left\{F_{e, k^{\prime}, 0}: k^{\prime}<k\right\} \cup F_{e, k, s}\right|,
$$


and for all $(2 e+k)$-states $\nu$,

there exists an $x \in F_{e, k, s}$ such that $\nu(2 e+k, x, s)=\nu$

iff

there exists an $\hat{y} \in \hat{F}_{e, k, s}$ such that $\nu(2 e+k, \hat{y}, s)=\nu$

where $\nu(2 e+k, x, s)$ is measured w.r.t. $\left\{U_{n, s}\right\}_{n, s<\omega}$ and $\left\{\hat{V}_{n, s}\right\}_{n, s<\omega}$ and $\nu(2 e+k, \hat{y}, s)$ is measured w.r.t. $\left\{\hat{U}_{n, s}\right\}_{n, s<\omega}$ and $\left\{V_{n, s}\right\}_{n, s<\omega}$. (More regarding how we will ensure this later.) Let $x \in F_{e, k, \infty}$ iff for all $s, x \in F_{e, k, s}$, and let $\hat{y} \in \hat{F}_{e, k, \infty}$ iff for all $s, \hat{y} \in \hat{F}_{e, k, s}$.

Since both $F_{e, k, s}$ and $\hat{F}_{e, k, s}$ are finite and nonincreasing (in size) in $s$, for all $(2 e+k)$-states $\nu$, there exists an $x \in F_{e, k, \infty}$ such that $\nu(2 e+k, x)=$ $\nu$ (w.r.t. $\left\{U_{n}\right\}_{n<\omega}$ and $\left\{\hat{V}_{n}\right\}_{n<\omega}$ ) iff there exists a $\hat{y} \in \hat{F}_{e, k, \infty}$ such that $\nu(2 e+k, \hat{y})=\nu$ (w.r.t. $\left\{V_{n}\right\}_{n<\omega}$ and $\left.\left\{\hat{U}_{n}\right\}_{n<\omega}\right)$. Hence there is an isomorphism between $\bigcup\left\{F_{e, k, \infty}: e, k<\omega\right\}$ and $\bigcup\left\{\hat{F}_{e, k, \infty}: e, k<\omega\right\}$ (For this to occur it is important that we meet (2) with respect to $(2 e+k)$-states or at least $(\max (e, k))$-states rather than just $k$-states. As a referee noted it is possible to replace $(2 e+k)$-states with $(\max (e, k))$-states in (2).) Should it be the case (it will not) that for all $e, \bigcup\left\{F_{e, k, \infty}: e, k<\omega\right\}=M_{e}$ and $\bigcup\left\{\hat{F}_{e, k, \infty}: e, k<\omega\right\}=\hat{M}_{e}$, we will have met $\left(R_{\nu}\right)$.

The easiest way to meet condition (2) is to use matching. That is, for all $x \in F_{e, k, s}$, ensure that $\hat{x} \in \hat{F}_{e, k, s}$ and $\nu(2 e+k, x, s)=\nu(2 e+k, \hat{x}, s)$, where $\hat{x}$ is the copy of $x$ in $\hat{\omega}, \nu(2 e+k, x, s)$ is measured w.r.t. $\left\{U_{n, s}\right\}_{n, s<\omega}$ and $\left\{\hat{V}_{n, s}\right\}_{n, s<\omega}$, and $\nu(2 e+k, \hat{x}, s)$ is measured w.r.t. $\left\{\hat{U}_{n, s}\right\}_{n, s<\omega}$ and $\left\{V_{n, s}\right\}_{n, s<\omega}$ (i.e., $x \in U_{i, s}$ iff $\hat{x} \in \hat{U}_{i, s}$, and $x \in \hat{V}_{i, s}$ iff $\hat{x} \in V_{i, s}$. For more on the use of matching see [CDS].) However, we cannot use matching and meet condition (1) (using matching implies that $\left|F_{e, k, s}\right|=\left|\hat{F}_{e, k, s}\right|$ ).

Very informally, we will use the following strategy to meet condition (2): At every stage $s$, associated with every $F_{e, k, s}$ and $\hat{F}_{e, k, s}$, there will be a $(2 e+k)$ state $\nu_{e, k, s}$. Assume there is an $\hat{x} \in \hat{F}_{e, k, s}$ such that $\hat{x} \in V_{n, s+1}-V_{n, s}$. If $\nu(2 e+k, \hat{x}, s)=\nu \neq \nu_{e, k, s}$, then we will ensure that there is at least one element of $F_{e, k, s}$ in the same $(2 e+k)$-state $\nu$ and will raise the least one to match $\hat{x}$ 's new state. If $\nu(2 e+k, \hat{x}, s)=\nu_{e, k, s}$ and there are at least two elements of $F_{e, k, s}$ in the same $(2 e+k)$-state $\nu_{e, k, s}$, we will raise one of them to match $\hat{x}$ 's new state. Otherwise we will define $F_{e, k, s+1} \subset F_{e, k, s}$ and $\hat{F}_{e, k, s+1} \subset \hat{F}_{e, k, s}$ so that all the elements in these sets have the same $(2 e+k)$-state $\nu_{e, k, s+1}$ at stage $s+1$ and condition (1) holds. Hopefully these sets will be big enough for us to continue using this strategy and maintain (1). (We will take a similar action if there is an $x \in F_{e, k, s}$ such that $x \in U_{n, s+1}-$ $U_{n, s}$.) We will call this strategy pseudomatching. (We will explore the details of pseudomatching later.)

Now if $\lambda \hat{x} \lim _{s \rightarrow \omega}\{e\}(\hat{x}, s)$ is a permutation then by (1) for large enough $s$ there exists a $\hat{z} \in \hat{F}_{e, k, s}$ such that $\{e\}(\hat{z}, s) \notin \bigcup\left\{F_{e, k^{\prime}, s}: k^{\prime} \leq k\right\}$. Hence, we can add $\{e\}(\hat{z}, s)$ to $U_{2 e}$ to meet $\left(P_{e, k}\right)$ without interfering with (2) for $k^{\prime} \leq k$. However, if $\{e\}(\hat{z}, s) \in F_{e, k^{\prime}, s}$, this will interfere with condition (2) and the need to keep $\hat{U}_{2 e} \cap F_{e, k^{\prime}, \infty}=\varnothing$, for some $k^{\prime}>k$. To get around this problem, we will do the following: Assume we add $\{e\}(\hat{z}, s)$ to $U_{2 e}$ at stage $t$. We will define $F_{e, k^{\prime}, t}=\varnothing$ and $\hat{F}_{e, k^{\prime}, t}=\varnothing$, for all $k<k^{\prime} \leq t$. Hence, this 
will injure $P_{e, k^{\prime}}$, but if $\lambda \hat{x} \lim _{s \rightarrow \omega}\{e\}(\hat{x}, s)$ is a permutation, this will only injure finitely many $P_{e, k^{\prime}}$. (Again, we will go into more detail later.)

Let $T=\left\{x: x \in F_{e, k, s}-F_{e, k, s+1}\right.$, for some $\left.e, k, s<\omega\right\}$ and $\tilde{T}=\{\hat{x}: \hat{x} \in$ $\hat{F}_{e, k, s}-\hat{F}_{e, k, s+1}$ for some $\left.e, k, s<\omega\right\}$. Hence $\bigcup\left\{F_{e, k, \infty}: e, k<\omega\right\}=\bar{T}$ and $\bigcup\left\{\hat{F}_{e, k, \infty}: e, k<\omega\right\}=\tilde{T}$. Therefore, by the use of pseudomatching, there is an isomorphism between $\bar{T}$ and $\overline{\tilde{T}}$. We now need to extend this isomorphism into an automorphism. To do this, we will use the Extension Theorem. Hence $\left(R_{\nu}\right)$ will divide into two subrequirements, namely, $\exists^{\infty} x \in T$ with final $e$-state $\nu$ w.r.t. to $\left\{U_{n}\right\}_{n<\omega}$ and $\left\{\hat{V}_{n}\right\}_{n<\omega}$

$\exists^{\infty} \hat{x} \in \overline{\tilde{T}}$ with final $e$-state $\nu$ w.r.t. to $\left\{\hat{U}_{n}\right\}_{n<\omega}$ and $\left\{V_{n}\right\}_{n<\omega}$,

and

$$
\exists^{\infty} x \in T \text { with final } e \text {-state } \nu \text { w.r.t. to }\left\{U_{n}\right\}_{n<\omega} \text { and }\left\{\hat{V}_{n}\right\}_{n<\omega}
$$

iff

$\exists^{\infty} \hat{x} \in \tilde{T}$ with final $e$-state $\nu$ w.r.t. to $\left\{\hat{U}_{n}\right\}_{n<\omega}$ and $\left\{V_{n}\right\}_{n<\omega}$.

We will meet $\left(R_{\nu}^{0}\right)$ by using pseudomatching and meet $\left(R_{\nu}^{1}\right)$ by using the Extension Theorem. Before we state the Extension Theorem, the following definitions are needed.

Definition 5. Given states $\nu=\langle e, \sigma, \tau\rangle$ and $\nu^{\prime}=\left\langle e^{\prime}, \sigma^{\prime}, \tau^{\prime}\right\rangle$, then

(i) $\nu \preceq \nu^{\prime}$ iff $e \leq e^{\prime}, \sigma=\sigma^{\prime} \cap\{0,1, \ldots, e\}$ and $\tau=\tau^{\prime} \cap\{0,1, \ldots, e\}$,

(ii) $\nu^{\prime}$ covers $\nu\left(\nu \leq \nu^{\prime}\right)$ iff $e=e^{\prime}, \sigma \subseteq \sigma^{\prime}$, and $\tau^{\prime} \subseteq \tau$, and

(iii) $\nu^{\prime}$ co-covers $\nu$ iff $\nu \geq \nu^{\prime}$.

Definition 6. Given recursive enumerations $\left\{X_{s}\right\}_{s<\omega}$ and $\left\{Y_{s}\right\}_{s<\omega}$ of $X$ and $Y$,

(i) $X \backslash Y=\left\{z:(\exists s) z \in X_{s}-Y_{s}\right\}$, and

(ii) $X \searrow Y=(X \backslash Y) \cap Y$.

Theorem 7 (The Extension Theorem [So1, So2, XV.6]). Assume $T$ and $\tilde{T}$ are infinite r.e. sets and $\left\{U_{n}\right\}_{n<\omega},\left\{\tilde{V}_{n}\right\}_{n<\omega},\left\{\tilde{U}_{n}\right\}_{n<\omega}$, and $\left\{V_{n}\right\}_{n<\omega}$ are recursive arrays of r.e. sets. Let $\left\{T_{s}\right\}_{s<\omega}, \quad\left\{\tilde{T}_{s}\right\}_{s<\omega},\left\{U_{n, s}\right\}_{n, s<\omega},\left\{\tilde{V}_{n, s}\right\}_{n, s<\omega}$, $\left\{\tilde{U}_{n, s}\right\}_{n, s<\omega}$, and $\left\{V_{n, s}\right\}_{n, s<\omega}$ be a simultaneous enumeration of the above r.e. sets. For each full e-state $\nu$ define

$$
\begin{array}{r}
D_{\nu}^{T}=\left\{x: \exists s\left(x \in T_{s}-T_{s-1} \text { and } \nu=\nu(e, x, s)\right.\right. \\
\text { w.r.t. } \left.\left.\left\{U_{n, s}\right\}_{n, s<\omega} \text { and }\left\{\tilde{V}_{n, s}\right\}_{n, s<\omega}\right)\right\}
\end{array}
$$

and

$$
\begin{aligned}
D_{\nu}^{\widetilde{T}}=\left\{\hat{x}: \exists s\left(\hat{x} \in \tilde{T}_{s}-\tilde{T}_{s-1} \text { and } \nu=\nu(e, \hat{x}, s)\right.\right. \\
\text { w.r.t. } \left.\left.\left\{\tilde{U}_{n, s}\right\}_{n, s<\omega} \text { and }\left\{V_{n, s}\right\}_{n, s<\omega}\right)\right\} .
\end{aligned}
$$

If $x \in D_{\nu}^{T}$, we say that $\nu$ is the entry e-state of $x$ (likewise for $\hat{x}$ ). Suppose our simultaneous enumeration satisfies the following conditions:

$$
\begin{gathered}
\forall n\left[T \searrow \tilde{V}_{n}=\tilde{T} \searrow \tilde{U}_{n}=\varnothing\right], \\
(\forall \nu)\left[D_{\nu}^{\tilde{T}} \text { is infinite } \Rightarrow\left(\exists \nu^{\prime} \geq \nu\right)\left[D_{\nu^{\prime}}^{T} \text { is infinite }\right]\right], \\
(\forall \nu)\left[D_{\nu}^{T} \text { is infinite } \Rightarrow\left(\exists \nu^{\prime} \leq \nu\right)\left[D_{\nu^{\prime}}^{\tilde{T}} \text { is infinite }\right]\right] .
\end{gathered}
$$


(We will sometimes say that condition (4) is " $T$ covers $\tilde{T}$ " and condition (5) is " $\tilde{T}$ co-covers $T$ ".) Then there are r.e. sets $\hat{U}_{n}$ extending $\tilde{U}_{n}$ and $\hat{V}_{n}$ extending $\tilde{V}_{n}$ such that $R_{\nu}^{1}$ is satisfied. Furthermore, $\tilde{U}_{n}=\hat{U}_{n} \backslash \tilde{T}$ and $\tilde{V}_{n}=\hat{V}_{n} \backslash T$ (this follows from the proof since if $x \notin \tilde{U}_{n}$, we will only add $x$ to $\hat{U}_{n}$ if $x \in \tilde{T})$ and there are recursive functions $f_{2}$ and $g_{2}$ such that $\hat{U}_{n}={ }^{*} W_{f_{2}(n)}$ and $\hat{V}_{n}={ }^{*} W_{g_{2}(n)}$.

If we have a simultaneous enumeration of $T, \tilde{T},\left\{U_{n}\right\}_{n<\omega},\left\{\tilde{V}_{n}\right\}_{n<\omega}$, $\left\{V_{n}\right\}_{n<\omega}$, and $\left\{\tilde{U}_{n}\right\}_{n<\omega}$ that satisfies the hypotheses of the Extension Theorem and the following requirements:

$$
\begin{gathered}
\exists^{\infty} x \in \bar{T} \text { with final } e \text {-state } \nu \text { w.r.t. to }\left\{U_{n}\right\}_{n<\omega} \text { and }\left\{\tilde{V}_{n}\right\}_{n<\omega} \\
\text { iff } \\
\exists^{\infty} \hat{x} \in \tilde{T} \text { with final } e \text {-state } \nu \text { w.r.t. to }\left\{\tilde{U}_{n}\right\}_{n<\omega} \text { and }\left\{V_{n}\right\}_{n<\omega},
\end{gathered}
$$

then using the Extension Theorem it is possible to meet the $R_{\nu}$ 's (and clearly the $Q_{e}$ 's).

Recall that $\left\{M_{i}: i<\omega\right\}$ is a uniformly recursive collection of disjoint infinite recursive sets such that for all $x$ there exists an $i$ with $x \in M_{i}\left(\hat{M}_{i}\right.$ will be a copy of $M_{i}$ in $\left.\hat{\omega}\right)$. Assume that $\left\{U_{2 n+1, s}\right\}_{n, s<\omega}$ is a simultaneous enumeration of $\left\{W_{e}\right\}_{e<\omega},\left\{V_{2 n+1, s}\right\}_{n, s<\omega}$ is a simultaneous enumeration of $\left\{W_{e}\right\}_{e<\omega}$, and $\left\{V_{2 n, s}\right\}_{n, s<\omega}$ is a simultaneous enumeration of $\left\{M_{e}\right\}_{e<\omega}$ such that $U_{2 n+1}=W_{n}, V_{2 n+1}=W_{n}, V_{2 n}=\hat{M}_{n}$, and for every stage $s>0$ there exists a unique $\langle x, i\rangle$ such that either $i$ is odd and $x \in U_{i, s}-U_{i, s-1}$, or $\hat{x} \in V_{i, s}-V_{i, s-1}(\hat{x}$ is the copy of $x$ in $\hat{\omega})$.

We need to build a simultaneous enumeration $\left\{T_{s}\right\}_{s<\omega}, \quad\left\{\tilde{T}_{s}\right\}_{s<\omega}$, $\left\{U_{2 n, s}\right\}_{n, s<\omega},\left\{\tilde{V}_{n, s}\right\}_{n, s<\omega}$, and $\left\{\tilde{U}_{n, s}\right\}_{n, s<\omega}$ of $T, \tilde{T},\left\{U_{2 n}\right\}_{n<\omega},\left\{\tilde{V}_{n}\right\}_{n<\omega}$, and $\left\{\tilde{U}_{n}\right\}_{n<\omega}$ such that $\left(P_{e, k}\right)$ and $\left(\tilde{R}_{\nu}^{0}\right)$ are met and the enumeration satisfies the hypotheses of the Extension Theorem. (Clearly, if desired, the above enumerations can be combined into one simultaneous enumeration which also satisfies the hypotheses of the Extension Theorem.) Note that $T$ and $\tilde{T}$ will be automorphic r.e. sets; $\Phi(T)=\tilde{T}$.

For the rest of the construction, we will fix the following notation. We will use $\nu(e, x, s)$ for the full $e$-state of $x$ at stage $s$ with respect to $\left\{U_{n, s}\right\}_{n, s<\omega}$ and $\left\{\tilde{V}_{n, s}\right\}_{n, s<\omega}$ and $\nu(e, \hat{x}, s)$ for the full $e$-state of $\hat{x}$ at stage $s$ with respect to $\left\{\tilde{U}_{n, s}\right\}_{n, s<\omega}$ and $\left\{V_{n, s}\right\}_{n, s<\omega} . \hat{x}$ is the copy of $x$ in $\hat{\omega}$ and $x$ is the copy of $\hat{x}$ in $\omega$.

We will use pseudomatching to meet $\left(\tilde{R}_{\nu}^{0}\right)$ as follows: First, if $\hat{z} \notin \tilde{T}_{s}$, then there exists $e, k$ such that $\hat{z} \in \hat{F}_{e, k, s}$ (the same holds for $x \notin T_{s}$ ). Assume $\hat{z} \in\left(V_{n, s+1} \cap \tilde{T}_{s}\right)-V_{n, s}$ (where $\left.n \leq 2 e+k\right)$. Let $x$ be an element of $F_{e, k, s}$ such that $\nu(2 e+k, x, s)=\nu(2 e+k, \hat{z}, s)$ (such an element will always exist, see Lemma 8(i)). There are two cases, namely: If $\nu(2 e+k, x, s) \neq \nu_{e, k, s}$ or there are at least two different such $x$, place the least such $x$ into $\tilde{V}_{n}$ at Stage $s+1$.

Otherwise, define $L(s)$ and $\hat{L}(s)$ as any of the largest possible sets such that for some $(2 e+k)$-state $\nu_{e, k, s+1} \neq \nu_{e, k, s}, L(s)=\{y: \nu(2 e+k, y, s)=$ $\left.\nu_{e, k, s+1} \wedge y \in F_{e, k, s}\right\}$ and $\hat{L}(s)=\left\{\hat{w}: \nu(2 e+k, \hat{w}, s)=\nu_{e, k, s+1} \wedge \hat{w} \in\right.$ $\left.\hat{F}_{e, k, s}\right\}$. These sets will be big enough for us to continue pseudomatching 
and maintain (1) (for more see Lemma 8(viii)). We will define $F_{e, k, s+1} \subset L(s)$ and $\hat{F}_{e, k, s+1} \subset \hat{L}(s)$ such that (1) holds. For all $y \in F_{e, k, s}-F_{e, k, s+1}$ we will add $y$ to $T$ at stage $s+1$, and for all $\hat{w} \in \hat{F}_{e, k, s}-\hat{F}_{e, k, s+1}$ we will add $\hat{w}$ to $\tilde{T}$ at stage $s+1$. (To make life easier, we will always put one quarter of the integers in $\hat{L}(s)$ into $\tilde{T}$ and three quarters of those in $L(s)$ into $T$ at stage $s$. We have passed the problem of "matching" $\hat{z}$ on to the Extension Theorem.) We say that we were forced to dump for $e$ and $k$ at stage $s+1$ by $\hat{z}$ and we will call this action dumping for $e$ and $k$.

Assume $x \in\left(U_{n, s+1} \cap \bar{T}_{s}\right)-U_{n, s}$ (for $\left.n \leq 2 e+k\right)$. Let $\hat{z}$ be the least integer in $\hat{F}_{e, k, s}$ such that $\nu(2 e+k, x, s)=\nu(2 e+k, \hat{z}, s)$ (again such a $\hat{z}$ will always exist). If either $\nu(2 e+k, x, s) \neq \nu_{e, k, s}$ or there is a $y \in F_{e, k, s}$ such that $x \neq y$ and $\nu(2 e+k, y, s)=\nu_{e, k, s}$, then $\hat{z} \in \tilde{U}_{n, s+1}$. Otherwise, we will use the above dumping procedure for $e$ and $k$.

To meet $\left(P_{e, k}\right)$ we will take the following action at stage $s$. We will associate with $\left(P_{e, k}\right)$ a function $t(e, k, s)$. Initially $t(e, k, 0)=0$. Assume $\lambda \hat{x} \lim _{s \rightarrow \omega}\{e\}(\hat{x}, s)$ is a permutation and, for all $\hat{z} \in \hat{F}_{e, k, s},\{e\}_{s}(\hat{z}, t(e, k, s)) \downarrow$. If there exists $\hat{z}, \hat{w} \in \hat{F}_{e, k, s}$ such that $\hat{z} \neq \hat{w}$ but $\{e\}_{s}(\hat{z}, t(e, k, s))=$ $\{e\}_{s}(\hat{w}, t(e, k, s))$ then let $t(e, k, s+1)=t(e, k, s)+1$. If for some $\hat{z} \in$ $\hat{F}_{e, k, s},\{e\}(\hat{z}, t(e, k, s)) \notin \hat{V}_{2 e, s} \cup T_{s}$, then let

$$
t(e, k, s+1)=t(e, k, s)+1 \text {. }
$$

[If this is the case for infinitely many $k$ and for almost all $s$, then since pseudomatching ensures that $\hat{F}_{e, k, \infty} \subset V_{2 e} \cup \tilde{T}={ }^{*} \Phi\left(\tilde{V}_{2 e} \cup T\right) \neq^{*} p^{-1}\left(\tilde{V}_{2 e} \cup T\right)$, we do not need to take any more action to meet $\left(P_{e}\right)$, where $p(\hat{x})=\lim _{s \rightarrow \omega}\{e\}(\hat{x}, s)$ (see Lemma 9(iv) for more).] Otherwise, by (1) (see Lemma 8(viii)), there exists a $\hat{z} \in \hat{F}_{e, k, s}$ such that $\{e\}(\hat{z}, t(e, k, s)) \notin \bigcup\left\{F_{e, k^{\prime}, s}: k^{\prime} \leq k\right\}$. We will add $\{e\}(\hat{z}, t(e, k, s))$ to $U_{2 e}$ at stage $s+1$; let

$$
t(e, k, s+1)=t(e, k, s)+1 \text {; }
$$

and for all $k^{\prime}$ such that $k<k^{\prime} \leq s$, let $F_{e, k^{\prime}, s+1}=\varnothing$ and $\hat{F}_{e, k^{\prime}, s+1}=\varnothing$, if $x \in \bigcup\left\{F_{e, k^{\prime}, s}: k<k^{\prime} \leq s\right\}$ place $x$ into $T$ at stage $s+1$, and if $\hat{y} \in$ $\bigcup\left\{\hat{F}_{e, k^{\prime}, s}: k<k^{\prime} \leq s\right\}$ place $\hat{y}$ into $\tilde{T}$ at stage $s+1$. We say $k^{\prime}$ was $e$ injured by $k$, for all $k^{\prime}$ such that $k<k^{\prime} \leq s$. Since $\lambda \hat{x} \lim _{s \rightarrow \omega}\{e\}(\hat{x}, s)$ is a permutation, this action for one fixed $k$ can only $e$-injure finitely many $k^{\prime}$. (If $\lambda \hat{x} \lim _{s \rightarrow \omega}\{e\}(\hat{x}, s)$ is not a permutation, we may have infinitely many such $e$-injuries.) Note the action for $\left(P_{e, k}\right)$ will not injure any $P_{e^{\prime}, k^{\prime}}$, for any $e^{\prime} \neq e$ and for any $k^{\prime}$.

If we only add numbers to the $\tilde{U}_{n}$ 's and $\tilde{V}_{n}$ 's in this fashion, we will meet condition (3) of the Extension Theorem. By condition (2) and the way we add integers to $T$ and $\tilde{T}$, there is an $x$ such that $x \in\left(T_{s+1} \cup F_{e, k, s}\right)-T_{s}$ and $\nu(2 e+k, x, s)=\nu$ iff there is a $\hat{z}$ such that $\hat{z} \in\left(\tilde{T}_{s+1} \cup \hat{F}_{e, k, s}\right)-$ $\tilde{T}_{s}$ and $\nu(2 e+k, \hat{z}, s) \geq \nu$. Hence we will be able to meet conditions (4) and (5) of the Extension Theorem. Since both $F_{e, k, s}$ and $\hat{F}_{e, k, s}$ are finite and nonincreasing in $s$, for all $(2 e+k)$-states, there exists an $x \in F_{e, k, \infty}$ such that $\nu(2 e+k, x)=\nu$ iff there exists a $\hat{y} \in \hat{F}_{e, k, \infty}$ such that $\nu(2 e+k, \hat{y})=$ $\nu$. Hence, pseudomatching will meet $\left(\tilde{R}_{\nu}^{0}\right)$. If $x \in \tilde{V}_{2 e, s+1}-\tilde{V}_{2 e, s}$, then, by pseudomatching, $x \in F_{e, k, s+1} \subseteq M_{e}$. Thus $\tilde{V}_{2 e} \subseteq M_{e}$. 
THE CONSTRUCTION OF $T, \tilde{T},\left\{U_{2 n}\right\}_{n<\omega},\left\{\tilde{U}_{n}\right\}_{n<\omega},\left\{\tilde{V}_{n}\right\}_{n<\omega}$,

$$
\left\{F_{e, k, s}\right\}_{e, k, s<\omega} \text {, AND }\left\{\hat{F}_{e, k, s}\right\}_{e, k, s<\omega}
$$

Stage 0. Let $T_{0}=\tilde{T}_{0}=\varnothing$ and $U_{2 n, 0}=\tilde{U}_{n, 0}=\tilde{V}_{n, 0}=\varnothing$, for all $n \in \omega$. Let $t(e, k, 0)=0$, for all $e$ and $k$. Let $\nu_{e, k, 0}=\langle(2 e+k), \varnothing, \varnothing\rangle$. We will now define, by induction on $k, F_{e, k, 0}$ and $\hat{F}_{e, k, 0}$.

Let $m(e, 0)=1$ and $m(e, k)=\left|\bigcup\left\{F_{e, k^{\prime}, 0}: k^{\prime}<k\right\}\right|$ for $k>0$. Let $p(e, k)=2(2 e+k+1)$ (this is the maximum number of times we can dump for $e$ and $k$; see Lemma $8(\mathrm{v}))$ and $q(e, k)=2^{2(2 e+k+1)}$ (the number of $(2 e+k)$ states). Choose $F_{e, k, 0}$ as the first (in terms of order) $m(e, k)(4 q(e, k))^{p(e, k)}$ elements of $M_{e}-\bigcup\left\{F_{e, k^{\prime}, 0}: k^{\prime}<k\right\}$ and $\hat{F}_{e, k, 0}$ as the first $3 m(e, k)$ $\cdot(4 q(e, k))^{p(e, k)}$ elements of $\hat{M}_{e}-\bigcup\left\{\hat{F}_{e, k^{\prime}, 0}: k^{\prime}<k\right\}$.

(Let $n(e, k, s)$ be the number of times we have dumped for $e$ and $k$ before stage $s$. Fix some $e$ and $k$. Assume that $k$ is never $e$-injured by some $k^{\prime}<k$. In Lemma 8, we will show by induction on $s$ that

$$
\begin{aligned}
& a=\left|F_{e, k, s}\right| \geq m(e, k)(4 q(e, k))^{(p(e, k)-n(e, k, s))}, \\
& b=\left|\hat{F}_{e, k, s}\right| \geq 3 m(e, k)(4 q(e, k))^{(p(e, k)-n(e, k, s))},
\end{aligned}
$$

and that $b-a>m(e, k)$. Hence condition (1) always holds.)

Stage $s+1$. Unless otherwise defined below all parameters remain the same from stage $s$ to stage $s+1$.

Step 1 (Building $U_{2 e}$ and meeting $\left(P_{e, k}\right)$ ). For all $e \leq s$ and $k \leq s$, if $\{e\}_{s}(\hat{z}, t(e, k, s)) \downarrow$ for all $\hat{z} \in \hat{F}_{e, k, s}$, do the first case which applies.

Case a. If either $\hat{F}_{e, k, s}=\varnothing$; there exist $\hat{z}, \hat{w} \in \hat{F}_{e, k, s}$ such that $\hat{z} \neq \hat{w}$ and $\{e\}(\hat{z}, t(e, k, s))=\{e\}(\hat{w}, t(e, k, s))$; there exists a $\hat{z} \in \hat{F}_{e, k, s}$ such that $\{e\}(\hat{z}, t(e, k, s)) \in U_{2 e, s}$; or for some $\hat{z} \in \hat{F}_{e, k, s},\{e\}(\hat{z}, t(e, k, s)) \notin$ $\tilde{V}_{2 e, s} \cup T_{s}$; then let $t(e, k, s+1)=t(e, k, s)+1$.

Case b. Otherwise, by (1) (see Lemma 8(viii)), there exists a $\hat{z} \in \hat{F}_{e, k, s}$ such that $\{e\}(\hat{z}, t(e, k, s)) \notin \bigcup\left\{F_{e, k^{\prime}, s}: k^{\prime} \leq k\right\}$. We will add $\{e\}(\hat{z}, t(e, k, s))$ to $U_{2 e}$ at stage $s+1 ; t(e, k, s+1)=t(e, k, s)+1$; for all $k^{\prime}$ such that $k<k^{\prime} \leq s$, let $F_{e, k^{\prime}, s+1}=\varnothing$; if $x \in \bigcup\left\{F_{e, k^{\prime}, s}: k<k^{\prime} \leq s\right\}$, place $x$ into $T$ at stage $s+1$; and if $\hat{y} \in \bigcup\left\{\hat{F}_{e, k^{\prime}, s}: k<k^{\prime} \leq s\right\}$ place $\hat{y}$ into $\tilde{T}$ at stage $s+1$. We say $k^{\prime}$ was $e$-injured by $k$ at stage $s$, for all $k^{\prime}$ such that $k<k^{\prime} \leq s$.

(Note that $\{e\}(\hat{z}, t(e, k, s)) \in \hat{V}_{2 e, s} \cup T_{s}$. Therefore, if $\{e\}(\hat{z}, t(e, k, s)) \notin$ $T_{s}$ then $\{e\}(\hat{z}, t(e, k, s)) \in F_{e, k^{\prime}, s}$ for some $s \geq k^{\prime}>k$ and hence $\left.\{e\}(\hat{z}, t(e, k, s)) \in T_{s+1}\right)$.

Step 2 (Pseudomatching to meet $\left(\tilde{R}_{\nu}^{0}\right)$ ). Let $\langle x, i\rangle$ be such that either $i$ is odd and $x \in U_{i, s+1}-U_{i, s}$ or $\hat{x} \in V_{i, s+1}-V_{i, s}$. Use the first case which applies.

Case a. $x \in F_{e, k, s}, i \leq 2 e+k, k$ has not been $e$-injured by stage $s+1$, $x \in U_{i, s+1}-U_{i, s}$, and either $\nu(2 e+k, x, s) \neq \nu_{e, k, s}$ or there exists $y \neq x$ such that $y \in F_{e, k, s}$ and $\nu(2 e+k, y, s)=\nu_{e, k, s}$. Then let $\hat{z}$ be the least element of $\hat{F}_{e, k, s}$ such that $\nu(2 e+k, x, s)=\nu(2 e+k, \hat{z}, s)$ (such a $\hat{z}$ will always exist by (2); see Lemma 8(i)). Place $\hat{z}$ into $\tilde{U}_{i, s+1}$. 
Case b. $x \in F_{e, k, s}, i \leq 2 e+k, k$ has not been $e$-injured by stage $s+1$, $x \in U_{i, s+1}-U_{i, s}, \nu(2 e+k, x, s)=\nu_{e, k, s}$, and there do not exist $y \neq x$ such that $y \in F_{e, k, s}$ and $\nu(2 e+k, y, s)=\nu_{e, k, s}$. Let $L(s)$ be any of the largest possible sets such that for some $(2 e+k)$-state $\nu_{e, k, s+1} \neq \nu_{e, k, s}, L(s)=\{y$ : $\left.\nu(2 e+k, y, s)=\nu_{e, k, s+1} \wedge y \in F_{e, k, s}\right\}$. Let $\hat{L}(s)=\{\hat{w}: \nu(2 e+k, \hat{w}, s)=$ $\left.\nu_{e, k, s+1} \wedge \hat{w} \in \hat{F}_{e, k, s}\right\}$. Let $n$ and $j<4$ be such that $4 n+j=|L(s)|=|\hat{L}(s)|$ (by Lemma 8(ii) we can assume $|L(s)|=|\hat{L}(s)|$, and as to why $n \neq 0$, see Lemma 8(viii)). Let $F_{e, k, s+1}$ be the first (in terms of order) $n$ elements of $L(s)$, and let $\hat{F}_{e, k, s+1}$ be the first $3 n$ elements of $\hat{L}(s)$. For all $y \in F_{e, k, s+1}-$ $F_{e, k, s}$ we will add $y$ to $T$ at stage $s+1$, and for all $\hat{w} \in \hat{F}_{e, k, s+1}-\hat{F}_{e, k, s}$ we will add $\hat{w}$ to $\tilde{T}$ at stage $s+1$. Note that $x \notin F_{e, k, s+1}$. (We were forced to dump for $e$ and $k$ by $x$.)

Case c. $\hat{x} \in \hat{F}_{e, k, s}, i \leq 2 e+k, k$ has not been $e$-injured by stage $s+$ $1, \hat{x} \in V_{i, s+1}-V_{i, s}$, and either $\nu(2 e+k, \hat{x}, s) \neq \nu_{e, k, s}$ or there exist $y, z$ such that $y \neq z, \nu_{e, k, s}=\nu(2 e+k, z, s)=\nu(2 e+k, y, s), y \in$ $F_{e, k, s}$, and $z \in F_{e, k, s}$. Then let $z$ be the least element of $F_{e, k, s}$ such that $\nu(2 e+k, \hat{x}, s)=\nu(2 e+k, z, s)$. Place $z$ into $\tilde{V}_{i, s+1}$.

Case d. $\hat{x} \in \hat{F}_{e, k, s}, i \leq 2 e+k, k$ has not been $e$-injured by stage $s+1$. $\hat{x} \in V_{i, s+1}-V_{i, s}, \nu(2 e+k, \hat{x}, s)=\nu_{e, k, s}$, and there do not exist $y, z$ such that $y \neq z . \nu_{e, k, s}=\nu(2 e+k, z, s)=\nu(2 e+k, y, s), y \in F_{e, k, s}$, and $z \in F_{e, k, s}$. Define $L(s)$ as any of the largest possible sets such that for some $(2 e+k)$-state $\nu_{e, k, s+1} \neq \nu_{e, k, s}, L(s)=\left\{y: \nu(2 e+k, y, s)=\nu_{e, k, s+1} \wedge y \in F_{e, k, s}\right\}$. Define $\hat{L}(s)=\left\{\hat{w}: \nu(2 e+k, \hat{w}, s)=\nu_{e, k, s+1} \wedge \hat{w} \in \hat{F}_{e, k, s}\right\}$. Let $n$ and $j<4$ be such that $4 n+j=|L(s)|=|\hat{L}(s)|$ (again by Lemma 8(ii) we can assume $|L(s)|=|\hat{L}(s)|$, and as to why $n \neq 0$, again see Lemma 8(viii)). Let $F_{e, k, s+1}$ be the first $n$ elements of $L(s)$, and let $\hat{F}_{e, k, s+1}$ be the first $3 n$ elements of $\hat{L}(s)$. For all $y \in F_{e, k, s+1}-F_{e, k, s}$ we will add $y$ to $T$ at stage $s+1$, and for all $\hat{w} \in \hat{F}_{e, k, s+1}-\hat{F}_{e, k, s}$ we will add $\hat{w}$ to $\tilde{T}$ at stage $s+1$.

\section{THE VERIFICATION}

Lemma 8. For all $e, k, s$, and all $2 e+k$-states $\nu$, let $n(e, k, s)$ be the number of times we have dumped for $e$ and $k$ by the end of stage $s$ and assume $k$ is never e-injured by any $k^{\prime}\left(\right.$ for $\left.k^{\prime}<k\right)$. Let $\nu_{e, k, s}=\left\langle 2 e+k, \sigma_{s}, \tau_{s}\right\rangle$ Then:

(i) there exists an $x \in F_{e, k, s}$ such that $\nu(2 e+k, x, s)=\nu$ iff there exists a $\hat{y} \in \hat{F}_{e, k, s}$ such that $\nu(2 e+k, \hat{y}, s)=\nu$ (hence condition (2) holds);

(ii) for all $(2 e+k)$-states $\nu$, if $\nu=\nu_{e, k, s}$ then

$\left|\left\{\hat{z} \in \hat{F}_{e, k, s}: \nu(2 e+k, \hat{z}, s)=\nu\right\}\right|>\left|\left\{x \in F_{e, k, s}: \nu(2 e+k, x, s)=\nu\right\}\right|$, and if $\nu \neq \nu_{e, k, s}$ then

$\left|\left\{\hat{z} \in \hat{F}_{e, k, s}: \nu(2 e+k, \hat{z}, s)=\nu\right\}\right|=\left|\left\{x \in F_{e, k, s}: \nu(2 e+k, x, s)=\nu\right\}\right| ;$

(iii) for all $x \in F_{e, k, s}$, if $\nu(2 e+k, x, s)=\langle 2 e+k, \sigma, \tau\rangle$ then $\sigma \supseteq \sigma_{s}$ and $\tau \supseteq \tau_{s}$

(iv) for all $\hat{z} \in \hat{F}_{e, k, s}$, if $\nu(2 e+k, \hat{z}, s)=\langle 2 e+k, \sigma, \tau\rangle$ then $\sigma \supseteq \sigma_{s}$ and $\tau \supseteq \tau_{s}$

(v) $n_{s}=p(e, k)-n(e, k, s) \geq 0($ recall $p(e, k)=2(2 e+k+1))$; 
(vi) $a_{s}=\left|F_{e, k, s}\right| \geq m(e, k)(4 q(e, k))^{n_{s}}$ (recall that $q(e, k)=2^{2(2 e+k+1)}$, $m(e, 0)=1$, and $m(e, k)=\left|\bigcup\left\{F_{e, k^{\prime}, 0}: k^{\prime}<k\right\}\right|$, for $\left.k>0\right)$;

(vii) $b_{s}=\left|\hat{F}_{e, k, s}\right| \geq 3 m(e, k)(4 q(e, k))^{n_{s}}$; and

(viii) $b_{s}-a_{s}>m(e, k)$ (hence condition (1) holds).

Proof. We will prove (i)-(viii) simultaneously by induction on $s$. Clearly (i)(viii) hold at stage $0\left(\nu_{e, k, 0}=\langle 2 e+k, \varnothing, \varnothing\rangle\right.$. Assume the lemma holds for $s$. We will show that it holds for $s+1$. There are two cases. First assume we do not dump for $e$ and $k$ at stage $s+1$. When an element of $F_{e, k, s}$ changes its state and is not dumped, then exactly one element of $\hat{F}_{e, k, s}$ also changes its state to match (see Step 2, Case a). The same holds for $\hat{F}_{e, k, s}$ (see Step 2, Case c). Since $F_{e, k, s+1}=F_{e, k, s}$ and $\hat{F}_{e, k, s+1}=\hat{F}_{e, k, s}$, (i)-(viii) hold.

Assume we dump for $e$ and $k$ at stage $s+1$. Then $v_{e, k, s+1}$ is the state of all the elements in $L(s), \hat{L}(s), F_{e, k, s+1}$, and $\hat{F}_{e, k, s+1}$. We have that either $\sigma_{s+1} \supset \sigma_{s}$ and $\tau_{s+1} \supseteq \tau_{s}$ or $\sigma_{s+1} \supseteq \sigma_{s}$ and $\tau_{s+1} \supset \tau_{s}$ (by (iii), (iv), and Step $2)$. Think of the set of $(2 e+k)$-states as a partial order with $\left\langle 2 e+k, \sigma_{1}, \tau_{1}\right\rangle \leq$ $\left\langle 2 e+k, \sigma_{2}, \tau_{2}\right\rangle$ iff $\sigma_{1} \subseteq \sigma_{2}$ and $\tau_{1} \subseteq \tau_{2}$. A maximal chain has length at most $2(2 e+k+1)+1$. Therefore, we can only dump for $e$ and $k$ at most $2(2 e+k+1)$ times, and hence $(\mathrm{v})$ holds. Since $a_{s}=\left|F_{e, k, s}\right| \geq m(e, k)(4 q(e, k))^{n_{s}}$, it follows that $|L(s)| \geq 4 m(e, k)(4 q(e, k))^{n_{s+1}} \quad\left(n_{s+1}=n_{s}-1\right.$ and recall $q(e, k)$ is the number of $(2 e+k)$-states). Let $n$ and $j<4$ be such that $4 n+j=|L(s)|=$ $|\hat{L}(s)|$. Since $\left|F_{e, k, s+1}\right|=n,\left|\hat{F}_{e, k, s+1}\right|=3 n$ and $n \geq m(e, k)(4 q(e, k))^{n_{s+1}} \neq$ 0 .

It is very easy to show that for all $s, F_{e, k, s} \supseteq F_{e, k, s+1}, \hat{F}_{e, k, s} \supseteq \hat{F}_{e, k, s+1}$, $x \notin T_{s}$ iff there exist an $e$ and $k$ such $x \in F_{e, k, s}$, and $\hat{z} \notin T_{s}$ iff there exist an $e$ and $k$ such that $\hat{z} \in \hat{F}_{e, k, s}$. By Lemma 8(i) and since both $F_{e, k, s}$ and $\hat{F}_{e, k, s}$ are finite and nonincreasing (in size) in $s$, for all $(2 e+k)$-states, there exists an $x \in F_{e, k, \infty}$ such that $\nu(2 e+k, x)=\nu$ iff there exists a $\hat{y} \in \hat{F}_{e, k, \infty}$ such that $\nu(2 e+k, \hat{y})=\nu$. Hence, $\Phi\left(U_{e}\right)=\tilde{U}_{e}$ and $\Phi^{-1}\left(V_{e}\right)=\tilde{V}_{e}$ is an isomorphism between $\bigcup\left\{F_{e, k, \infty}: e, k<\omega\right\}=\bar{T}$ and $\bigcup\left\{\hat{F}_{e, k, \infty}: e, k<\right.$ $\omega\}=\tilde{T}$. Hence we have met $\left(R_{\nu}^{0}\right)$.

Clearly, for all $n, T \searrow \tilde{V}_{n}=\tilde{T} \searrow \tilde{U}_{n}=\varnothing$, and hence condition (3) of the Extension Theorem holds. Assume $x \in T_{s+1}-T_{s}$. So there exist an $e$ and $k$ such that $x \in F_{e, k, s}$. By Lemma 8(i), there exists a $\hat{z} \in \hat{F}_{e, k, s}$ such that $\nu(2 e+k, x, s)=\nu(2 e+k, \hat{z}, s)$. Now by either Step 2, Case b; Step 2, Case d; or Step 1, Case b, $\hat{z} \in \tilde{T}_{s+1}-\tilde{T}_{s}$ and $\nu(2 e+k, x, s+1) \geq \nu(2 e+k, \hat{z}, s+1)$. Since $F_{e, k, s}$ is finite and nonincreasing in $s$, for all $\nu, D_{\nu}^{T}$ is infinite implies there exists a $\nu^{\prime} \leq \nu$ such that $D_{\nu^{\prime}}^{\tilde{T}}$ is infinite, and hence condition (5) of the Extension Theorem holds. Similar reasoning shows that condition (4) of the Extension Theorem holds. Therefore, $\Phi\left(U_{e}\right)=\hat{U}_{e}$ and $\Phi^{-1}\left(V_{e}\right)=\hat{V}_{e}$ is an effective automorphism and $\Phi(T)=\tilde{T}$.

We will now show $\left(P_{e}\right)$ is met.

Lemma 9. Assume that $\lambda \hat{x} \lim _{s \rightarrow \omega}\{e\}(\hat{x}, s)$ is a permutation (otherwise, $\left(P_{e}\right)$ is already met). For all $k$ :

(i) for all $m$, there is an $s_{m}$ such that $t\left(e, k, s_{m}\right)>m$;

(ii) there is a stage $s$ such that, after stage $s, k$ never e-injures any $k^{\prime}>k$; 
(iii) there is an $l>k$ such that $F_{e, l, \infty} \neq \varnothing$ and $\hat{F}_{e, l, \infty} \neq \varnothing$; and

(iv) $\left(P_{e}\right)$ is met.

Proof. (i) Assume that for some stage $s^{\prime}, t\left(e, k, s^{\prime}\right)=m$. Let $s>s^{\prime}$ be such that $s \geq k, e$, and for all $\hat{z} \in \hat{F}_{e, k, 0},\{e\}_{s}(\hat{z}, m) \downarrow$. If $t(e, k, s)=m$, at stage $s+1$ either Case a or Case b of Step 1 will apply. Therefore, $t(e, k, s+1)>m$.

(ii) Let $m$ be such that for all $\hat{z} \in \hat{F}_{e, k, 0}$, for all $s \geq m,\{e\}(\hat{z}, m)=$ $\{e\}(\hat{z}, s)$. Let $s \geq s_{m}$ be such that we never dump for $e$ and $k$ after stage $s$ ( $s$ exists by Lemma 8(v)). Step 1, Case b may only be applied once after stage $s$ (the action that causes the $e$-injuries only takes place in Step 1, Case b).

(iii) By Lemma 8(vi), Lemma 8(vii), and Step 1, Case b, we know that $F_{e, k^{\prime}, \infty}=\varnothing$ iff $\hat{F}_{e, k^{\prime}, \infty}=\varnothing$ iff $k^{\prime}$ was $e$-injured by some $k<k^{\prime}$. Therefore, $F_{e, 0, \infty} \neq \varnothing$. Once $k$ is $e$-injured, $k$ will never $e$-injure any $k^{\prime}>k$. If there are finitely many stages $s$ such that some $k e$-injures at stage $s$, then almost all $F_{e, k, \infty}$ and $\hat{F}_{e, k, \infty}$ are nonempty. Otherwise, there are infinitely many stages $s$ such that some $k e$-injures at stage $s$, but no $k_{1} \leq k \quad e$-injures at any stage greater than $s$. For each such $s, F_{e, s+1, \infty} \neq \varnothing$ and $\hat{F}_{e, s+1, \infty} \neq \varnothing$.

(iv) Let $p(\hat{x})=\lim _{s \rightarrow \omega}\{e\}(\hat{x}, s)$. There are two cases, namely: If there are infinitely many $\hat{x} \in \bigcup\left\{\hat{F}_{e, k, \infty}: k<\omega\right\}$ such that $p(\hat{x}) \in U_{2 e}$, then $\Phi\left(U_{2 e}\right)=$ $\hat{U}_{2 e} \neq^{*} p^{-1}\left(U_{2 e}\right)$. Otherwise, there exists an infinite subset $\hat{Z}$ of $\left(\bigcup\left\{\hat{F}_{e, k, \infty}\right.\right.$ : $\left.k<\omega\} \cap V_{2 e}\right)$ such that $p(\hat{Z}) \cap\left(\tilde{V}_{2 e} \cup T\right)=\varnothing$, and hence $\Phi\left(\hat{V}_{2 e} \cup T\right)={ }^{*}$ $\left(V_{2 e} \cup \tilde{T}\right) \neq^{*} p^{-1}\left(\tilde{V}_{2 e} \cup T\right)$.

Note that we have not produced two effectively automorphic r.e. sets $T$, $\tilde{T}$ such that for any automorphism $\Psi$ of $\mathscr{E}^{*}$ which can be induced by a $\Delta_{2}$ permutation $\Psi(T) \neq^{*} \tilde{T}$ (although $\Phi(T)=\tilde{T}$ and $\Phi$ is not induced by a $\Delta_{2}$ permutation, there may be another automorphism $\Psi$ such that $\Psi$ is induced by a $\Delta_{2}$-permutation and $\left.\Psi(T)={ }^{*} \tilde{T}\right)$. In fact, we believe that it may be the case that all of the known applications of the Extension Theorem to a standard skeleton have produced automorphisms or isomorphisms that are induced by $\Delta_{2}$-permutations. For example, consider Maass's result [Ma] that all co-infinite low promptly simple sets are effectively automorphic. We conjecture that these effective automorphisms can be induced by $\Delta_{2}$-permutations. However, we also conjecture that there is a modification of the standard Extension Machinery (as in [So2, XV.6]) so that the resulting automorphism or isomorphism cannot be induced by a $\Delta_{2}$-permutation and there are two effectively automorphic r.e. which are not automorphic by any automorphism of $\mathscr{E}^{*}$ induced by a $\Delta_{2}-$ permutation.

We will end with two questions. Is there an effective automorphism which is only induced by a permutation of degree $0^{\prime \prime}$ ? Is there a noneffective automorphism which is induced by a permutation whose degree is less than $0^{\prime \prime}$ ?

\section{REFERENCES}

[Ch] P. Cholak, Automorphisms of the lattice of recursively enumerable sets, Ph.D. Dissertation, University of Wisconsin, May, 1991.

[CDS] P. Cholak, R. Downey, and M. Stob, Automorphisms of the lattice of recursively enumerable sets: Promptly simple sets, Trans. Amer. Math. Soc. 332 (1992), 555-570.

[HS] L. Harrington and R. Soare, Post's program and incomplete recursively enumerable sets, Proc. Nat. Acad. Sci. U.S.A. 88 (1991), 10242-10246. 
[Ma] W. Maass, Recursively enumerable generic sets, J. Symbolic Logic 49 (1982), 809-823.

[Po] E. Post, Recursively enumerable sets of positive integers and their decision problems, Bull Amer. Math. Soc. 50 (1944), 248-316.

[So1] R. Soare, Automorphisms of the lattice of recursively enumerable sets, Part I: Maximal sets, Ann. of Math. (2) 100 (1974), 80-120.

[So2] - Recursively enumerable sets and degrees, Perspect. Math. Logic, Springer-Verlag, Berlin, Heidelberg, New York, and Tokyo, 1987.

Victoria University of Wellington, P.O. Box 600, Wellington, New Zealand

E-mail address, R. Downey: downey@math.vuw.ac.nz

Current address, P. Cholak: Department of Mathematics, Cornell University, Ithaca, New York 14853

E-mail address, P. Cholak: cholak@math.cornell.edu 